Network Working Group

Request for Comments: 2214

Category: Standards Track
F. Baker

Cisco Systems

J. Krawczyk

ArrowPoint Communications

A. Sastry

Cisco systems

September 1997

\title{
Integrated Services Management Information Base Guaranteed Service Extensions using SMIv2
}

\section{Status of this Memo}

This document specifies an Internet standards track protocol for the Internet community, and requests discussion and suggestions for improvements. Please refer to the current edition of the "Internet Official Protocol standards" (STD 1) for the standardization state and status of this protocol. Distribution of this memo is unlimited.

Abstract

This memo defines a portion of the Management Information Base (MIB) for use with network management protocols in TCP/IP-based internets. In particular, it defines objects for managing the the interface attributes defined in the Guaranteed Service of the Integrated Services Model. Comments should be made to the Integrated Services Working Group, intserveisi.edu.

Table of Contents

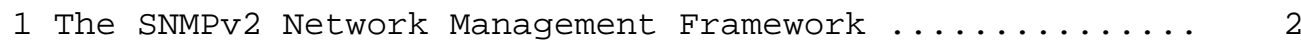

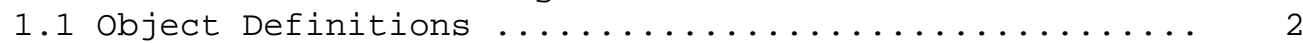

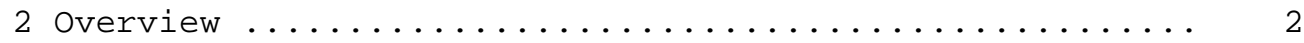

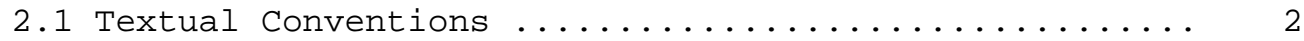

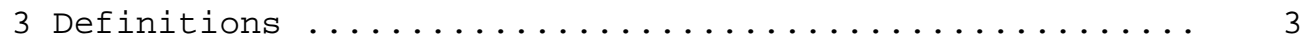

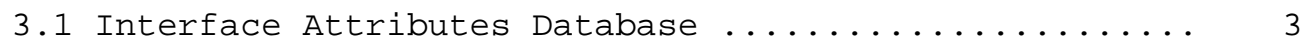

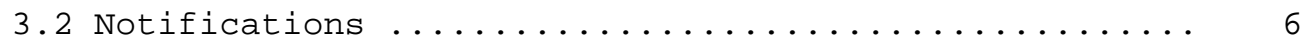

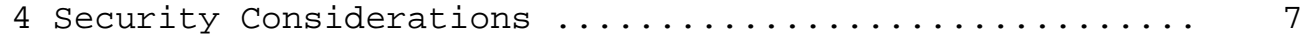

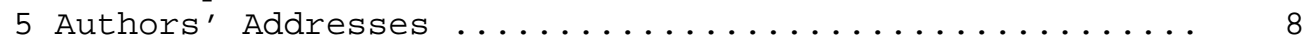

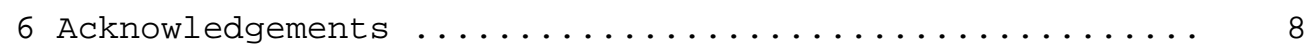

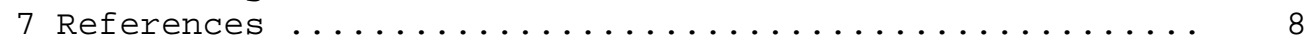


1. The SNMPv2 Network Management Framework

The SNMPv2 Network Management Framework consists of four major components. They are:

- RFC 1441 which defines the SMI, the mechanisms used for describing and naming objects for the purpose of management.

- STD 17, RFC 1213 defines MIB-II, the core set of managed objects for the Internet suite of protocols.

- RFC 1445 which defines the administrative and other architectural aspects of the framework.

o RFC 1448 which defines the protocol used for network access to managed objects.

The Framework permits new objects to be defined for the purpose of experimentation and evaluation.

\subsection{Object Definitions}

Managed objects are accessed via a virtual information store, termed the Management Information Base or MIB. Objects in the MIB are defined using the subset of Abstract Syntax Notation One (ASN.1) defined in the SMI. In particular, each object type is named by an OBJECT IDENTIFIER, an administratively assigned name. The object type together with an object instance serves to uniquely identify a specific instantiation of the object. For human convenience, we often use a textual string, termed the descriptor, to refer to the object type.

\section{Overview}

\subsection{Textual Conventions}

Several new data types are introduced as a textual convention in this MIB document. These textual conventions enhance the readability of the specification and can ease comparison with other specifications if appropriate. It should be noted that the introduction of the these textual conventions has no effect on either the syntax nor the semantics of any managed objects. The use of these is merely an artifact of the explanatory method used. Objects defined in terms of one of these methods are always encoded by means of the rules that define the primitive type. Hence, no changes to the SMI or the SNMP are necessary to accommodate these textual conventions which are adopted merely for the convenience of readers and writers in pursuit 
of the elusive goal of clear, concise, and unambiguous MIB documents.

3. Definitions

INTEGRATED-SERVICES-GUARANTEED-MIB DEFINITIONS : := BEGIN

IMPORTS

\begin{tabular}{|c|c|c|}
\hline MODULE-IDENTITY, O & BJECT-TYPE & FROM SNMPV2-SMI \\
\hline Rowstatus & & FROM SNMPV2-TC \\
\hline MODULE-COMPLIANCE, & OBJECT-GROUP & FROM SNMPV2-CONF \\
\hline intSrv & FROM & INTEGRATED-SERVICES-MIB \\
\hline ifIndex & & FROM IF-MIB; \\
\hline
\end{tabular}

-- This MIB module uses the extended OBJECT-TYPE macro as

-- defined in [9].

intSrvGuaranteed MODULE-IDENTITY

LAST-UPDATED "9511030500Z" -- Thu Aug 28 09:04:22 PDT 1997

ORGANIZATION "IETF Integrated Services Working Group" CONTACT-INFO

" $\quad$ Fred Baker

Postal: Cisco Systems

519 Lado Drive

Santa Barbara, California 93111

Tel: $\quad+18056810115$

E-Mail: fredecisco.com"

DESCRIPTION

"The MIB module to describe the Guaranteed Service of

the Integrated Services Protocol"

$::=\{$ intSrv 5$\}$

intSrvGuaranteedobjects

intSrvGuaranteedNotifications

intSrvGuaranteedConformance
OBJECT IDENTIFIER

$::=\{$ intsrvGuaranteed 1$\}$

OBJECT IDENTIFIER

$::=\{$ intSrvGuaranteed 2$\}$

OBJECT IDENTIFIER

$::=\{$ intSrvGuaranteed 3$\}$

\footnotetext{
-- The Integrated Services Interface Attributes Database

-- contains information that is shared with other reservation

-- procedures such as ST-II.

intSrvGuaranteedIfTable OBJECT-TYPE SYNTAX SEQUENCE OF IntSrvGuaranteedIfEntry

MAX-ACCESS not-accessible

STATUS current
}

Baker, et. al.

Standards Track

[Page 3] 


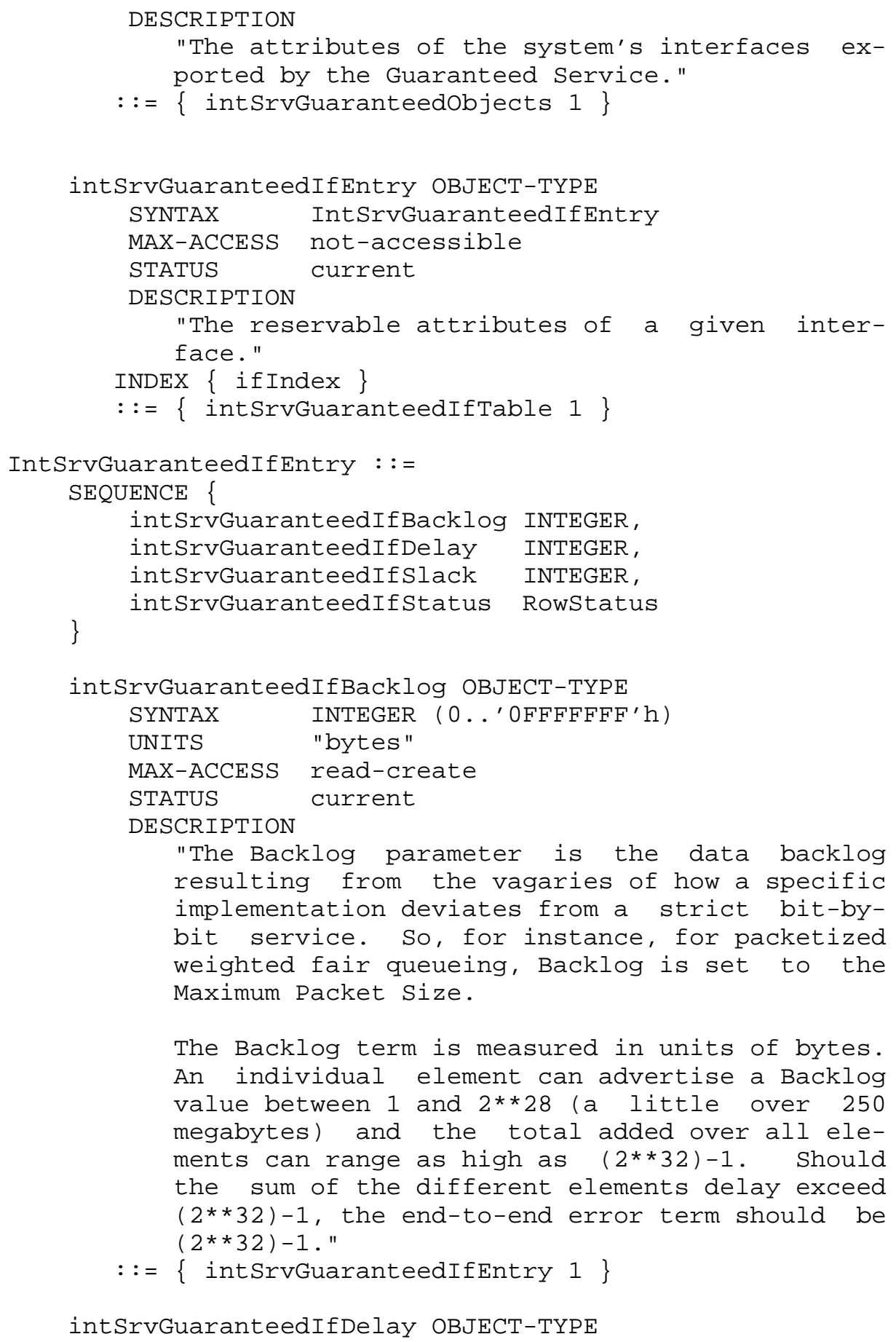




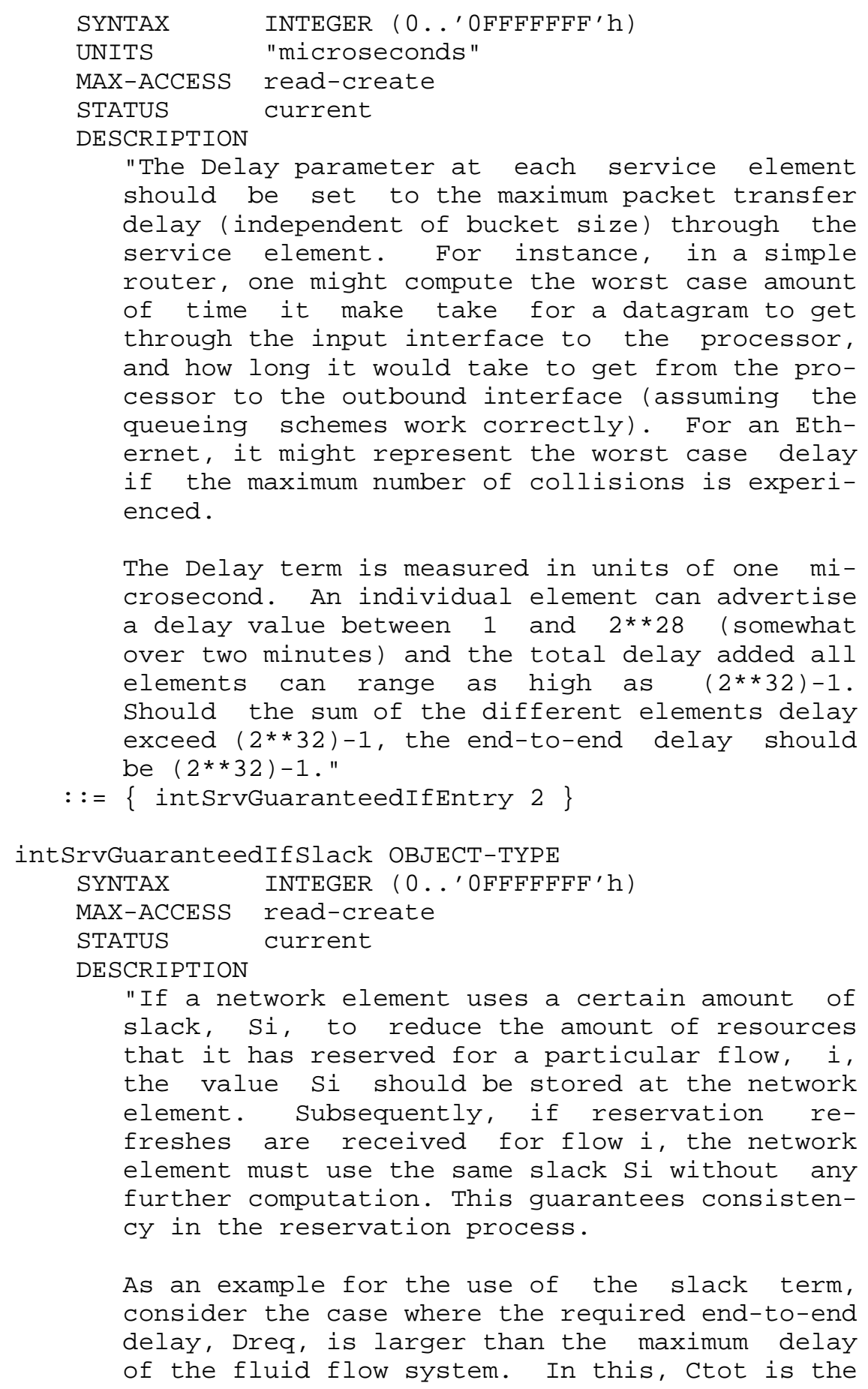




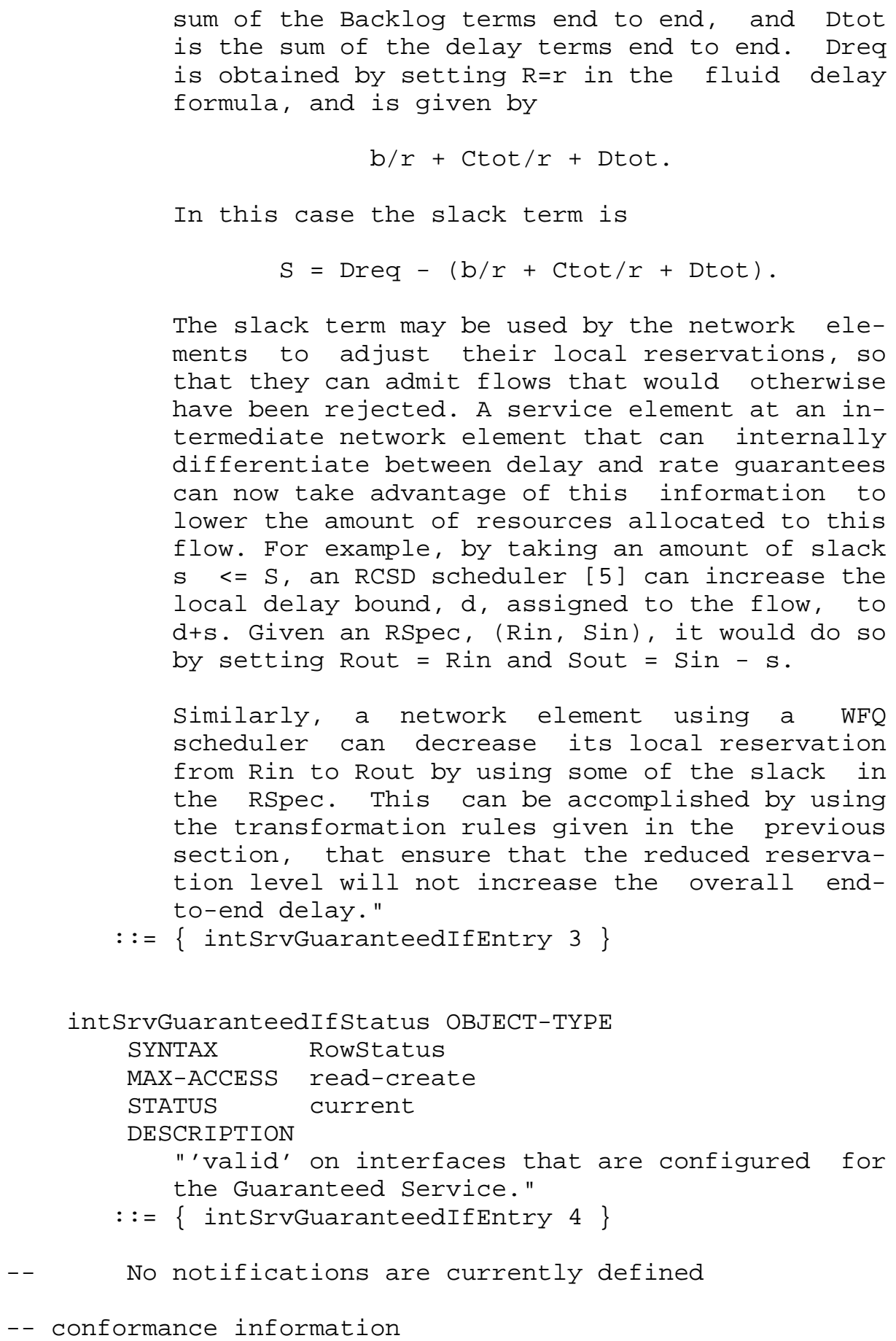




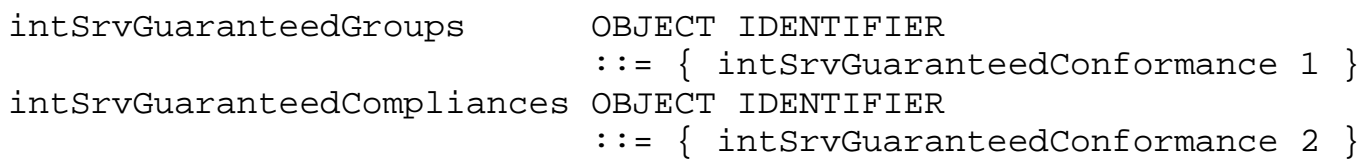

-- compliance statements

intSrvGuaranteedCompliance MODULE-COMPLIANCE

STATUS current

DESCRIPTION

"The compliance statement "

MODULE -- this module

MANDATORY-GROUPS \{

intSrvGuaranteedIfAttribGroup

\}

$::=\{$ intSrvGuaranteedCompliances 1$\}$

intSrvGuaranteedIfAttribGroup OBJECT-GROUP

OBJECTS \{

intSrvGuaranteedIfBacklog,

intSrvGuaranteedIfDelay,

intSrvGuaranteedIfSlack,

\}

intSrvGuaranteedIfStatus

STATUS current

DESCRIPTION

"These objects are required for systems supporting the Guaranteed Service of the Integrated Services Architecture."

$::=\{$ intSrvGuaranteedGroups 2$\}$

END

4. Security Considerations

The use of an SNMP SET results in an RSVP or Integrated Services reservation under rules that are different compared to if the reservation was negotiated using RSVP. However, no other security considerations exist other than those imposed by SNMP itself. 


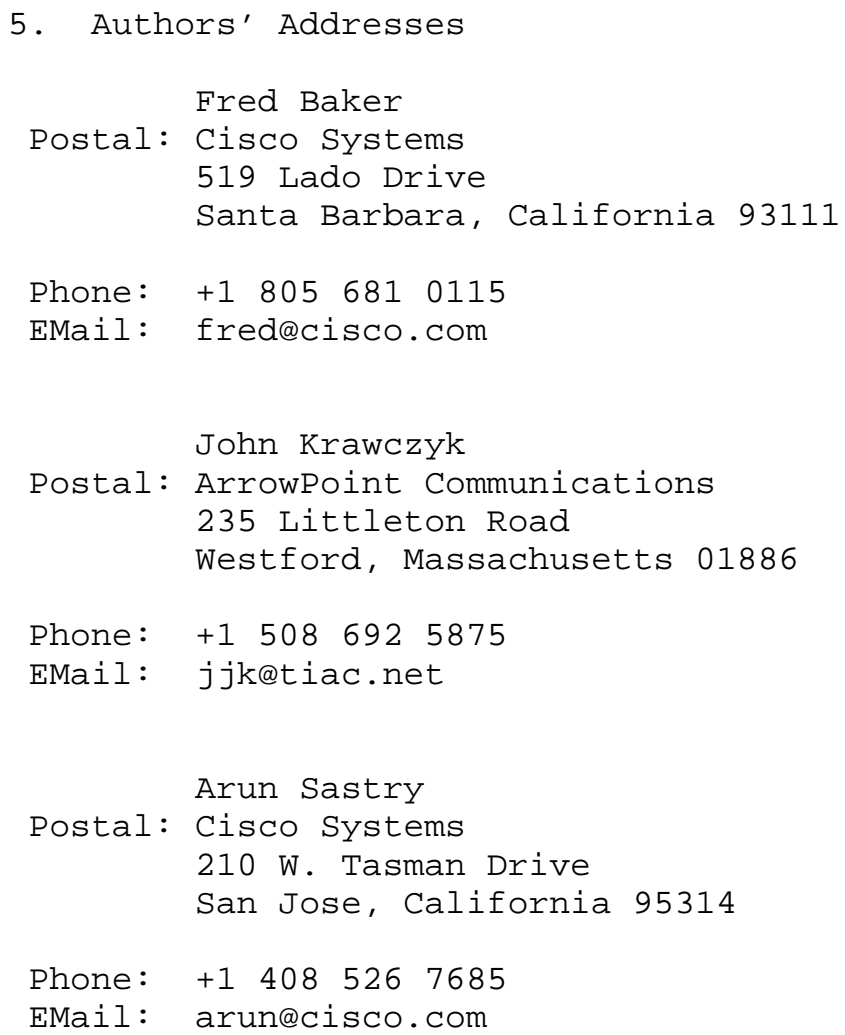

6. Acknowledgements

This document was produced by the Integrated Services Working Group.

7. References

[1] Rose, M., Editor, "Management Information Base for Network Management of TCP/IP-based internets", STD 17, RFC 1213, May 1990 .

[2] Information processing systems - Open systems Interconnection - Specification of Abstract Syntax Notation One (ASN.1), International Organization for Standardization. International Standard 8824, (December, 1987).

[3] Information processing systems - Open Systems Interconnection - Specification of Basic Encoding Rules for Abstract Notation One (ASN.1), International Organization for Standardization. International Standard 8825, (December, 1987). 
